\title{
The cytokine hypothesis: A neurodevelopmental explanation for the emergence of schizophrenia later in life
}

\author{
Julia Howard \\ Department of Molecular Microbiology and Immunology, Johns Hopkins University Bloomberg School of Public Health, Baltimore, \\ USA \\ Email: Jhoward@jhsph.edu
}

Received 20 June 2013; revised 20 July 2013; accepted 15 August 2013

Copyright (C 2013 Julia Howard. This is an open access article distributed under the Creative Commons Attribution License, which permits unrestricted use, distribution, and reproduction in any medium, provided the original work is properly cited.

\begin{abstract}
There is increasing evidence for the cytokine hypothesis, which states that exposure to elevated cytokines in utero due to maternal immune activation is a major risk factor for the development of schizophrenia later in life. This is supported by numerous epidemicologic studies that connect multiple infections with schizophrenia emergence. Furthermore, cytokines are critically involved in early neurodevelopment and deviations from the norm can result in abnormal neuroanatomy and brain chemistry. Animal models of schizophrenia also support the critical role of developmental neuroinflammation in predisposing the brain to anatomical and behavioral abnormalities. Although there is strong evidence for the critical role of cytokines, they most likely work with other contributing risk factors such as genetic predisposition. New evidence indicates that cytokine exposure in utero may prime the brain and that a second stressor during adolescence, referred to as a second hit, may activate existing developmental vulnerabilities resulting in the emergence of clinical schizophrenia. Further knowledge of these pathogenic processes and risk factors could be very instrumental in reducing risk and slowing emergence of schizophrenia.
\end{abstract}

Keywords: Schizophrenia; Cytokines; Inflammation; Neurodevelopment

\section{INTRODUCTION}

Schizophrenia, a disease that causes lifelong disability affects about $1 \%$ of the world's population [1,2]. People of all ethnic backgrounds, genders, socioeconomic statuses, and nationalities are affected, although prevalence is higher among males [1,3].

Although the strong genetic component of schizophre- nia has been well established, with a concordance rate of $45 \%$ among monozygotic twins, it is clear that environmental factors contribute significantly to the risk as well [4,5]. Researchers have demonstrated that environmental contributions can induce compromised brain circuitry and chemistry, especially early in life [6]. This can set the stage for irregular developmental processes in the central nervous system (CNS) and ultimately lead to schizophrenia [7].

Research also indicates that cytokines can play a prominent role in altering the course of normal brain maturation. The cytokine hypothesis postulates that prenatal exposure to pro-inflammatory cytokines due to maternal immune activation can cause abnormalities in fetal brain development. Support for this hypothesis has grown with the emergence of epidemiologic data, research on the role of cytokines in development, and use of animal models [2]. This paper will critically examine the support for the role of cytokines and explore emerging research to determine whether they can truly account for the development of this debilitating disorder.

\section{THE CYTOKINE HYPOTHESIS}

\subsection{Epidemiologic Support}

Epidemiologic evidence linking schizophrenia to maternal viral infection emerged as early as the 1950's, when a disproportionate number of children born after the influenza pandemic of 1957 developed schizophrenia in early adulthood [8]. In recent years, researchers have solidified these original observations with multiple epidemiologic studies that link young adult onset of schizophrenia with prenatal exposure to the influenza virus, particularly during the second trimester [9]. Using maternal serum samples drawn during pregnancy in the Child Health and Development Study (CHDS), researchers have found that offspring risk of developing schizophrenia increased up to 7 times if influenza exposure 
occurred during the first half of gestation, but there was no increased risk associated with influenza infection during the third trimester [10].

Similar trends have also been found with prenatal exposure to rubella, measles, polio, herpes simplex virus (HSV), Toxoplasma gondii, varicella zoster and diphtheria [2,9]. Maternal serum specimens from CHDS have revealed that elevated maternal $T$. gondii IgG antibodies are associated with a 2 fold increased risk of developing schizophrenia in offspring [10]. Maternal serum samples collected by the National Collaborative Perinatal Project (NCPP) revealed that elevated maternal antibodies against HSV-2 was significantly associated with offspring development of schizophrenia. Further, researchers found that risk was specifically related to activation of latent virus into lytic cycle or recent infection [4]. Prenatal infection with rubella increases risk of offspring developing schizophrenia by 10 - 20 times [5]. Given that prenatal exposure to many different pathogens (bacteria, parasites, and viruses) increase risk, researchers came to the conclusion that there must be common factors to the immune response that a number of pathogens are able to activate [10].

Epidemiologic data indicates that these common mediators are cytokines [2]. The CHDS cohort study showed that elevation of interleukin-8 (IL-8) during the second or third trimester put the offspring at increased risk of schizophrenia. They also found that when maternal tumor necrosis factor $\alpha(\mathrm{TNF} \alpha)$ was significantly elevated at birth, children had greater rates of developing psychosis [10]. These result support the clear role of cytokine exposure in utero as a risk factor.

\subsection{Cytokines During Pregnancy}

There is further evidence that cytokines can interact with the fetus through a number of ways. Cytokines are small enough that they can invade the fetal CNS by crossing the blood brain barrier (BBB) by active transport, through any disruptions in the BBB, or through circumventricular organs [11]. There is also evidence that cytokines can be produced by the placenta itself [5]. The fetus can also produce and secrete its own cytokines. Since a functional immune system does not develop until the third trimester, this capability is not seen until fairly late in gestation [2]. There is also evidence that cytokines can interact with the vagus nerve, which in turn will transmit signals via afferent nerve fibers to the brainstem. The extent and effects of this interaction are still unclear. Regardless of how cytokines access the fetal environment, studies show that interluekin 1 (IL-1), interluekin 6 (IL-6), and TNF $\alpha$ increase in the placenta in response to maternal immune activation, while only IL-6 and TNF $\alpha$ increase in the amniotic fluid [12]. Presence of cytokines in the fetal environment, certainly support the cyotkine hypothesis.

\subsection{Cytokines and the Neurodevelopment}

In addition to their immune function, cytokines are critically involved in neurodevelopment. In the fetal brain, cytokine receptors are expressed on many neuronal cell types and are involved in numerous processes of early neurodevelopment, which follows a tightly regulated pattern that is critical to efficient neural functioning in adulthood $[2,13]$.

Neurogenesis is the process by which neurons and other neural cell types are generated from progenitor cells. They will receive signals from several molecules, including cytokines that determine their developmental fate [12]. Specifically, IL-1 $\beta$ is involved in the conversion of midbrain progenitor cells into dopaminergic neurons, while interferon $\gamma$ (IFN $\gamma$ ) is involved in differentiation of cholinergic neural cell precursors $[7,14]$. Deviations in cytokine titer can result in abnormal neurogenesis, and an over or underabundance neural cell types.

Cytokines also play an important regulatory role in synaptogenesis, which is the formation of synapses between neurons to allow for efficient communication between cells throughout the nervous system. TNF $\alpha$ is involved in modulation of synaptic strength in the hippocampus and when depleted there is a decrease in cortical dendrite formation $[2,15]$. IL-1 $\beta$ enhances dopaminergic cell outgrowth specifically, while IL-6 inhibits outgrowth overall $[9,16]$. In rats, high concentrations of the three major pro-inflammatory cytokines (TNF $\alpha$, IL-6 and IL-1 $\beta$ ) decrease cortical dendrite formation, a hallmark of schizophrenia that also results in a decrease in synaptic connectivity $[2,17]$. Therefore, it is very clear that cytokines play a prominent role in regulating synapse formation during early development.

While, altered synaptic formation has effects on its own, it also influences pruning, which is the process by which cells that have made proper synaptic connections are maintained, while cells that do not enter neural networks undergo apoptosis [12]. Fewer synaptic connections can result in excessive pruning. Furthermore the pruning process is also regulated by cytokines. In vitro experiments demonstrated that pro-inflammatory cytokines decrease survival of fetal dopaminergic and serotinergic neurons and experiments have revealed that IL$1 \beta$ decreases neuron survival in the rat hippocampus [8]. Also, IL-6 is involved in apoptosis of fetal rat serotinergic neurons, but when paired with IL- $1 \beta$ in high concentrations, promotes survival of dopaminergic neurons. However, in low concentrations the two cytokines promote apoptosis of midbrain dopaminergic neurons. In early fetal development, TNF $\alpha$ plays a neurotrophic role to ventral mesencephalic dopaminergic neurons, but later 
becomes neurotoxic [2]. Interleukin 9 (IL-9) functions in autocrine and paracrine function to promote cell survival in the cortex [15]. Therfore, alterations in numerous cytokines can result in altered synaptogenesis and the disorganized neural networks often seen in schizophrenia [1].

Myelination, which is the process by which oligodendrocytes (a type of glial cell) wrap around neuronal axons creating insulation, is also controlled by cytokines. Myelination allows for quicker and more efficient neurotransmission [18]. In schizophrenia myelination is partially arrested due to low numbers of oligodendrocytes, which is the result excessive microglia activation caused by maternal immune activation $[17,18]$. Not only do microglia attack and destroy oligodendrocytes under the influence of TNF $\alpha$ and IFN $\gamma$, but TNF $\alpha$ also compromises oligodendrocyte growth and inhibits expression of genes encoding for myelin proteins [18].

On top of the numerous abnormalities in neuroanatomy, schizophrenia is accompanied by various changes in neurotransmission, many of which are immune driven. The dopaminergic system in particular, is very vulnerable to immune dysregulation, because neurogenesis, differentiation, and migration of dopaminergic neurons occur very early in development. Therefore, prenatal exposure to pro-inflammatory cytokines, especially IL-1 $\beta$, results in faulty density, distribution, and connectivity within the dopaminergic system that is irreversible [13]. Furthermore, IL-2 is a key modulator of dopamine metabolism, while IL-6 is also involved in synthesis of dopamine $[12,16]$.

The crucial role cytokines play during early brain development clearly supports the cyotkine hypothesis. Their extensive involvement in neurodevelopment makes it clear that any alterations in cytokines can permanently alter the course of neurodevelopment and can contribute to increased vulnerability to a complex disease like schizophrenia.

\subsection{Animal Models of Schizophrenia}

Additional support for the cytokine hypothesis is found in numerous animal models. Pregnant mice were injected with a strain of human influenza and although offspring showed no signs of infection upon birth, they later showed abnormalities in brain anatomy consistent with excessive pruning, decreased dendrite density, and abnormal migration seen in schizophrenia. As adults, the offspring also showed strange behavior patterns, such as a deficits in pre-pulse inhibition (PPI) to the acoustic startle response, which is representative of decreased sensory motor gating seen in schizophrenics. These behavioral deficits were reversed with acute administration of anti-psychotic drugs, confirming that the abnormal behavior was similar to clinical behavioral abnormalities shown in schizophrenics [9].

These results were confirmed in rhesus monkeys, prenatally exposed to influenza, who experienced extensive abnormalities post-puberty. This experiment was especially important, because cortical development is far more advanced in primates [7].

In another experiment, pregnant mice were treated with polyribocytidicilic acid (PolyI:C), a synthetic double stranded RNA that initiates an antiviral immune response, at a gestation day 9, which is equivalent to first trimester in people. This resulted in an antiviral-like immune response in the mother. Offspring experienced deficits in PPI, associative learning, and spatial working memory. In addition, mice were more sensitive to dopamine agonists and showed decreased dopamine receptors in the prefrontal cortex, which are indicative of irregulatarities in the dopaminergic mesolimbic system [2].

The same experiment was repeated at gestation day 17, a time corresponding to the second trimester. Behavioral abnormalities were less pronounced and offspring only showed notable impairment in reversal learning and spatial working memory. In addition, dopaminergic dysfunction was less apparent [2].

Reference [19] repeated the same experiment on a different set of mice 2 - 6 days after birth. In mice, immune and brain development occurring during the first two weeks of postnatal life correspond with the third trimester [20]. Abnormalities in behavior emerged in 10 12 weeks (post-puberty), but there were no significant impairments in dopamine systems. Mice showed increased anxiety and impairments in memory, social behavior, and sensory gating [21].

Comparison of these three experiments indicates that time of infection is of utmost importance and infection at different parts of pregnancy or early life confers different vulnerabilities that may ultimately result in different symptom clusters. Different areas of the brain develop at different times; therefore immune dysfunction can have varied developmental outcomes depending on what stage in development infection occurs [21]. Furthermore, considering that cytokines have different, even opposite effects depending on developmental stage, outcomes can vary immensely based on time [22].

When closely examining the differences between mice exposed during the equivalent of first trimester and second trimester, reference [2] revealed that a major difference between the two mice groups were the cytokines expressed. Exposure to PolyI:C during late gestation resulted in significantly higher concentrations of IL-10 in the fetal brain. In addition, IL-10 response balanced elevated IL-6, resulting in IL-6 to IL-10 ratios fairly close to control ratios [2]. IL-10 has been shown to support pregnancy and appears to play a protective role 
against some of the disruptive affects of pro-inflammatory cytokines [14]. Therefore, we might expect that upregulation of IL-10 may counteract some of the detrimental affects that result from increased IL-6. To test this theory, reference [2] preformed another experiment, following up PolyI:C treatment at gestation day 9 with IL-10 treatment. IL-10 treatment prevented the behavioral and neurochemical abnormalities seen with PolyI:C treatment alone [2]. However, subsequent experiments have shown that over expression of IL-10 can result in extensive behavioral abnormalities as well, indicating that IL-10 does not serve any protective function by itself. Rather, it seems that deviations away from the balance between pro-inflammatory and anti-inflammatory cytokines results in developmental abnormalities [23].

To ensure that cytokine imbalance was the cause of abnormal development resulting in schizophrenic-like symptoms in mice and not an effect of differential maternal care due to infection, reference [24] injected pregnant mice with PolyI:C or a vehicle treatment on day 9 of gestation. To measure the affects of post-natal maternal care, a group of offspring born to infected mothers were cross fostered and raised by an uninfected mother, and a group of offspring born to healthy dams were raised by a surrogate mother who had been infected. Regardless of maternal care, offspring who were prenatally exposed to PolyI:C developed a variety of dopaminergic and glutamatergic abnormalities as well as other neuroanatomical differences consistent with schizophrenia [24].

We have now established that there is substantial evidence for the critical role of cytokines found in epidemiologic data, studies of brain development and maturation, and results of animal models. Still, the hypothesis does not fully account for the long gap between neurodevelopmental insult and onset of symptoms, nor does it offer an explanation for any of the mechanistic changes [5]. The remainder of this paper will examine other modulators that might contribute to the dysfunction caused by cytokines and how these changes are carried out throughout the remainder of development.

\section{MODULATORS THAT ENHANCE CYTOKINE IMBALANCE}

Despite the strong evidence for the cytokine hypothesis, not everyone exposed to prenatal cytokine imbalance develops schizophrenia. Therefore, we must consider the fact that cytokines are probably not acting alone and numerous other factors must be considered in light of or in combination with cytokine effects.

\subsection{The Stress Response}

A major factor that must be considered is the stress re- sponse, which is closely tied to the immune system. The stress response can be activated by cytokines, resulting in activation of the hypothalamic-pituitary adrenal (HPA) axis, including upregulation of glucocorticoids [2]. Reference [25] found that elevated stress was a significant predictor of high IL-6 to IL-10 ratios in the $1^{\text {st }}$ trimester and elevated C Reactive Protein, which is a marker of systemic inflammation, in the $2^{\text {nd }}$ trimester. Individuals exposed to severe maternal stress prenatally often have robust cytokine and inflammatory responses as adults, resembling those of chronically stressed individuals even if the individual is not under stress at the time [3].

Animal models also support the connection between stress and the immune system. Injection of pregnant monkeys with adrenalcorticotropic hormone can alter the physiology and immune system of offspring. Also, monkeys exposed to synthetic corticosteroids in utero had altered thymus structure, indicating that prenatal stress altered the course of immune system development [3]. Therefore, stress can bolster the effects of early cytokine exposure.

\subsection{Prenatal Nutrition}

Cytokines are primarily responsible for the signs of sickness, which can result in fever, malaise, reduced social behavior, decreased food and water intake, and even weight loss [2]. Alterations in behavior may confer increased risk of schizophrenia independently of cytokines. After all, epidemiologic data indicates that schizophrenia risk is doubled by malnutrition, and maternal infection during the second trimester is highly associated with low birth weight, demonstrating that there is significant interaction between nutrition, immune function, and schizophrenia [14]. Caloric and fat intake, as well as glycemic index can all influence the inflammatory response as well. For example, omega 3 fatty acids are known to suppress inflammation and patients with low omega 3 intakes will develop a larger inflammatory response under stressful conditions [12]. Maternal undernutrition also has a strong affect on thymic and lymphoid tissue development [3]. Subsequently, prenatal nutrition must be considered as well.

\subsection{Sex Differences}

Additionally, sex-differences may confer differential risks due to inherent differences in development in the immune system or the nervous system. Schizophrenia symptoms are different between males and females and prevalence is notably higher among males [1]. These observations were confirmed when comparing male and female rats born to immune challenged mothers. Anatomical changes occurred earlier in male rats, and prefrontal cortex volume reduction was more accelerated in 
males. In addition, behavioral abnormalities emerged first and were more widespread in male rats [26]. Additional experiments revealed that PPI abnormalities were lowest during estrus in female rats. This is mirrored in women; symptoms of schizophrenia are most severe when estrogen is low [22]. These findings indicate that there is an interaction between sex, the CNS, and the immune system, that results in different outcomes of schizophrenia.

\subsection{Genetic Risk}

Beyond sex chromosomes, we cannot ignore the role of genetics in general. After all, most offspring who experience inflammation prenatally do not develop schizophrenia, indicating that there must be some type of predisposition that increases risk [25]. There are many candidate genes that have been identified as increasing risk of schizophrenia. Of 250 candidate genes, about 20\% are found to interact with influenza, herpes simplex virus 1 , or cytomegalovirus, while about $15 \%$ interact with rubella or T. gondii [5]. These interactions are indicative of infectious influence on gene expression, which can ultimately increase risk of schizophrenia. There are about 5 genes that are considered of particular importance.

Nuclear receptor related 1 (NURR1) is a transcription factor involved in differentiation of midbrain dopaminergic neurons. The nurr 1 mutation in combination with a stressful experience (such as postnatal isolation in mice or rats) can result in hyper locomotor activity, PPI deficits, and alterations in dopamine and serotonin metabolism consistent with schizophrenia [23].

The Neuregulin-1 (nrg1) gene encodes for a protein that interacts with receptor tyrosine-protein kinase erbB4 (ErbB4), which is expressed in high concentration on midbrain dopaminergic neurons. NRG1 is critically involved in neurodevelopment, specifically neuronal migration, synaptogenesis, and myelination [27]. Studies show that IL-1 $\beta$ interacts with NRG1, upregulating expression of $n r g 1$ and increasing cytokine release. This suggests that NRG1 activation by IL-1 $\beta$ enhances the pro-inflammatory response [25].

To test the role of NRG1 in neuropathology, reference [27] administered NRG1 to neonatal mice and found it activated ErbB4 and upregulated expression of tyrosine hydroxylase [27]. Tyrosine hydroxylase is the rate-limiting enzyme of dopamine synthesis and is a marker of pre-synaptic dopamine [13]. Increased tyrosine hydroxylase ultimately increased dopamine levels, and a hyperdopaminergic state was sustained in the medial prefrontal cortex into adulthood. Post puberty the mice began to show behavioral impairments in PPI, latent inhibition, social interactions. In humans with a nrg1 mutation, abnormal affects are only be seen after insult, such as traumatic brain injury or neonatal hypoxia [27].
Catechol-O-methyl transferase (COMT) is an enzyme that is responsible for catecholamine metabolism and plays an important role in clearance of dopamine from synapses in the cortex. Some risk is correlated with a phenotype that is less active, and therefore less efficient in regulating dopamine metabolism [23]. However, there is also risk associated with over activity of COMT and certain anti-psychotics actually decrease COMT levels [5].

The reln gene encodes for Reelin, which is a protein involved in migration and synaptogenesis [5]. It's expressed in high concentration during neurodevelopment, but remains active throughout life [7]. Post mortem studies of schizophrenics show that reln expression is reduced in certain brain areas [1].

Studies with the reeler mutant mouse show extensive issues in orientation and layering of neurons, indicating that the reln gene is crucial for organization of neurons and synaptogenesis. Furthermore, prenatal exposure to influenza alters reln expression in offspring, resulting in volume decreases consistent with schizophrenics and abnormal orientation of neurons [1].

Certain HLA haplotypes may also predispose immune dysregulation by initiating an excessive inflammatory response that can be triggered by prenatal immune exposure [23]. The HLA-G gene seems to be particularly important; not only does it serve a protective function to the placenta, but it is involved in resistance to lysis by natural killer cells. Natural killer cells can only kill trophoblast cells if HLA-G is blocked. HLA-G also regulates cytokine levels and can upregulate IL-3 and IL-1 $\beta$ and down-regulate TNF $\alpha$. During maternal immune activation, TNF $\alpha$ is increased, leading to reduced expression of HLA-G and increased activation of natural killer cells. This overabundance of natural killer cells can be detrimental to developing neural cells, which will quickly become targets without the protection of HLA-G [14].

There are certainly many genes that can introduce significant risk for developing schizophrenia. The majority of these genes are critically regulated by the immune system, signifying the important link between maternal immune activation, cytokines, and schizophrenia risk.

\section{THE TWO-HIT MODEL}

While the risk conferred by early cytokine exposure is fairly well established, the cytokine hypothesis still fails to explain the long gap between prenatal insult and the onset of symptoms almost twenty years later. The most popular way to explain this is with the two-hit model: genetic and/or environmental factors disrupt early neurodevelopment, which creates long term error. Exposure to a second hit can trigger the onset of schizophrenic symptoms, by activating underlying vulnerabilities in the 
CNS. Not only can this long-term vulnerability exist in the brain, but it can also exist in the immune system [6].

\subsection{Huntington's Disease as a Model}

While there is little epidemiological data tracking the presence of these early life abnormalities in individuals who will go on to develop schizophrenia, there is a fair amount of research based on Huntington's disease that can be used in parallel with some of the twin studies on schizophrenia. Although Huntington's disease differs from schizophrenia in many respects there are several key similarities. The disease is caused by an autosomal dominant mutation, but clinical Huntington's disease typically doesn't emerge for up to 40 years [28]. Onset is usually preceded by behavioral abnormalities, but the nature of these abnormalities differs with age. Onset in the 30's or 40's is associated with depression, while adolescent and early adult onset (that corresponds with the typical age of onset for schizophrenia) is usually associated with schizophreniform psychosis [29]. IL-6 and excessive inflammation contribute to the neurdegeneration that occurs with onset [28]. These observations suggest that inflammation may be a trigger for Huntington's disease too and that there is some vulnerability in early adulthood that makes psychosis a common side effect of inflammation within the brain. In addition, veterans who experienced brain injury were 5 times more likely to develop psychosis if the injury occurred before 20 years of age [29]. All data points to adolescence as a critical period of vulnerability for development of psychosis.

\subsection{Development during the Critical Period}

Although, the potential for schizophrenia is in place from an early age, there is some type of vulnerability during adolescence that allows it to be activated. There are several possibilities of what this vulnerability could be. First, brain development continues into the early 20's. Pruning occurs until about 20 years of age, while myelination occurs until about 25 years of age [17]. In addition, many areas of the cortex do not reach functional maturity until early adulthood [29]. Therefore, the initial hit can have long lasting effects on the course of developmental.

Additionally, development of the immune system into puberty is well documented. In adolescence, the immune system undergoes a series of changes, including a decline in B cell and T cell numbers and reduced proliferative responsiveness, and thymic volume continues to decrease [30].

In concordance with the cytokine hypothesis and the two hit model, researchers believe that exposure to a robust inflammatory response in utero, could result in alternative programming of the immune system such that prenatal inflammation permanently alters homeostasis of the immune system [25]. This may not become apparent until later in immune system development. Schizophrenia is associated with various neuroimmunological abnormalities, including chronic low-grade inflammation and overactive and sensitized microglia, indicating an altered baseline of immune homeostasis. This picture is supported by studies of mice exposed prenatally to influenza, PolyI:C, or IL-6 treatments. All of these treatment groups experienced enhanced microglia and persistent presence of pro-inflammatory cytokines in the periphery [7].

\subsection{The Second Hit}

Although much research needs to be completed on the second hit, researchers have found that it can be a number of stressors. There is increased risk of schizophrenia among individuals raised in urban compared to rural areas and this follows a dose-response curve such that a greater number of years lived in an urban area increases the risk of psychosis [31]. Research has also shown that excessive cannabis use can also provide the second hit [23]. Adolescents who are considered heavy cannabis users have a 6-fold increased risk of psychotic symptoms compared with non-users. Studies suggest that cannabis may interact with the COMT gene, which can result in alterations of dopamine circuits. Additionally, increased prevalence of childhood trauma, particularly emotional, physical or sexual abuse, which occurs before age 16 is associated with a 7-fold increase in development of psychotic symptoms [31].

Generally, it appears that any extreme stressor can provide a second hit, though it seems that the second hit must activate underlying neuroimmune and CNS abnormalities. In a recent experiment, prenatal immune activation with PolyI:C injection in rodents was followed up by various stressors, including electric foot shock, restraint stress, swimming stress and water deprivation, on alternate days during mouse puberty. Stress exposure lead to numerous behavioral abnormalities consistent with schizophrenia. These changes were not present prior to the stressor and applying the same stressors at a later period did not result in the same degree of abnormalities. Upon closer inspection, researchers found that the initial prenatal immune activation was sufficient to induce many changes, the exception being dopamine, which was only altered following the stressors during puberty. Additionally, the second stressor resulted in a 3-fold increase in microglia activation and an increase in IL- $1 \beta$ and TNF- $\alpha$, but there were no notable changes in cortisol within the CNS [32]. Therefore, it seems that the second hit can be anything that activates the CNS immune system, which initiates a hyper-response and ultimately unearths abnormalities built into the brain during early development. 


\section{CONCLUSION}

A wealth of research from epidemiologic, brain pathology, and animal research supports the role of cytokine imbalance as a source of abnormal neurodevelopment leading to schizophrenia. However, cytokine imbalance alone in the prenatal period is not enough to induce schizophrenia up to 20 years later and research indicates that there are many other factors at play. Genes, stress, and malnutrition can exacerbate the negative effects that cytokine imbalance has on neurodevelopment. Adolescence seems to be a particularly vulnerable period due to numerous changes that occur in brain and immune system development. Should a second hit occur during this critical period, normal developmental trajectories are further altered, leading to pathological changes that can result in the emergence of clinical schizophrenia. The second hit can be a number of environmental stressors, ranging from secondary infection to excessive cannabis use. However, little is known about these risk factors, but knowledge of them will be of critical importance. Not only would it allow us to interfere and possibly prevent emergence all together, but it could also provide us with invaluable information on treatment options that could not only treat symptoms but also prevent progression. Undoubtedly, having a deeper understanding of the emergence, pathogenesis, and progression of schizophrenia will be very valuable in combating this debilitating neurological disease.

\section{ACKNOWLEDGEMENTS}

First and foremost, I would like to acknowledge and thank Dr. Noel Rose for his guidance and for going above and beyond in offering support and direction as my advisor. Additionally, I would like to thank Dr. Sabra Klein and Dr. Alan Scott for their time and thoughtful comments. I would like to thank Dr. Linda Gorman who first taught me about schizophrenia and was also willing to offer her advice. I'd like to thank Dr. Joe Bressler for his thoughtful comments on environmental health and neuroimmunity.

\section{REFERENCES}

[1] Bayer, T.A., Falkai, P. and Maier, W. (1999) Genetic and non-genetic vulnerability factors in schizophrenia: The basis of the "two hit hypothesis". Journal of Psychiatric Research, 33, 543-548. doi:10.1016/S0022-3956(99)00039-4

[2] Meyer, U., Feldon, J. and Yee, B.K. (2009) A review of the fetal brain cytokine imbalance hypothesis of schizophrenia. Schizophrenia Bulletin, 35, 959-972. doi:10.1093/schbul/sbn022

[3] Kinney, D.K., Hintz, K., Shearer, E.M., Barch, D.H., Riffin, C., Whitley, K. and Butler, R. (2010) A unifying hypothesis of schizophrenia: Abnormal immune system development may help explain roles of prenatal hazards, post-pubertal onset, stress, genes, climate, infections, and brain dysfunction. Medical Hypotheses, 74, 555. doi:10.1016/j.mehy.2009.09.040

[4] Buka, S.L., Cannon, T.D., Torrey, E.F. and Yolken, R.H. (2008) Maternal exposure to herpes simplex virus and risk of psychosis among adult offspring. Biological Psychiatry, 63, 809-815. doi:10.1016/j.biopsych.2007.09.022

[5] Fatemi, S. H. and Folsom, T. D. (2009) The neurodevelopmental hypothesis of schizophrenia, revisited. Schizophrenia Bulletin, 35, 528-548. doi:10.1093/schbul/sbn187

[6] Maynard, T.M., Sikich, L., Lieberman, J.A. and LaMantia, A.S. (2001) Neural development, cell-cell signaling, and the "two-hit" hypothesis of schizophrenia. Schizophrenia Bulletin, 27, 457-476.

doi:10.1093/oxfordjournals.schbul.a006887

[7] Meyer, U. (2011) Developmental neuroinflammation and schizophrenia. Progress in Neuro-Psychopharmacology and Biological Psychiatry, 42, 20-34.

[8] Muller, N. and Schwarz, M.J. (2004) Role of the cytokine network in major psychoses. Advances in Molecular and Cell Biology, 31, 999-1032.

[9] Patterson, P.H. (2002) Maternal infection: window on neuroimmune interactions in fetal brain development and mental illness. Current Opinion in Neurobiology, 12, 115-118. doi:10.1016/S0959-4388(02)00299-4

[10] Brown, A.S. and Derkits, E.J. (2010) Prenatal infection and schizophrenia: A review of epidemiologic and translational studies. American Journal of Psychiatry, 167, 261-280. doi:10.1176/appi.ajp.2009.09030361

[11] Na, K.S., Jung, H.Y. and Kim, Y.K. (2012) The role of pro-inflammatory cytokines in the neuroinflammation and neurogenesis of schizophrenia. Progress in NeuroPsychopharmacology and Biological Psychiatry. doi:10.1016/j.pnpbp.2012.10.022

[12] Altamura, A., Pozzoli, S., Fiorentini, A. and Dell'Osso, B. (2012) Neurodevelopment and inflammatory patterns in schizophrenia in relation to pathophysiology. Progress in Neuro-Psychopharmacology and Biological Psychiatry.

[13] Meyer, U., Yee, B.K. and Feldon, J. (2007) The neurodevelopmental impact of prenatal infections at different times of pregnancy: The earlier the worse? The Neuroscientist, 13, 241-256. doi:10.1177/1073858406296401

[14] Debnath, M. and Chaudhuri, T.K. (2006) The role of HLA-G in cytokine homeostasis during early pregnancy complicated with maternal infections: A novel etiopathological approach to the neurodevelopmental understanding of schizophrenia. Medical Hypotheses, 66, 286-293. doi:10.1016/j.mehy.2005.06.033

[15] Deverman, B.E. and Patterson, P.H. (2009) Cytokines and CNS development. Neuron, 64, 61-78. doi:10.1016/j.neuron.2009.09.002

[16] Gaughran, F. (2002) Immunity and schizophrenia: Autoimmunity, cytokines, and immune responses. International Review of Neurobiology, 52, 275-302. doi:10.1016/S0074-7742(02)52013-4

[17] Bennett, M.R. (2011) Schizophrenia: Susceptibility genes, dendritic-spine pathology and gray matter loss. Progress 
in Neurobiology, 95, 275-300. doi:10.1016/j.pneurobio.2011.08.003

[18] Monji, A., Kato, T. and Kanba, S. (2009) Cytokines and schizophrenia: Microglia hypothesis of schizophrenia. Psychiatry and Clinical Neurosciences, 63, 257-265. doi:10.1111/j.1440-1819.2009.01945.x

[19] Anderson, G. and Maes, M. (2012) Schizophrenia: Linking prenatal infection to cytokines, the tryptophan catabolite (TRYCAT) pathway, NMDA receptor hypofunction, neurodevelopment and neuroprogression. Progress in Neuro-Psychopharmacology and Biological Psychiatry.

[20] Samuelsson, A.M., Jennische, E., Hansson, H.A. and Holmäng, A. (2006) Prenatal exposure to interleukin-6 results in inflammatory neurodegeneration in hippocampus with NMDA/GABAA dysregulation and impaired spatial learning. American Journal of Physiology-Regulatory, Integrative and Comparative Physiology, 290, R1345-R1356. doi:10.1152/ajpregu.00268.2005

[21] Ibi, D., Nagai, T., Kitahara, Y., Mizoguchi, H., Koike, H., Shiraki, A. and Yamada, K. (2009) Neonatal PolyI:C treatment in mice results in schizophrenia-like behavioral and neurochemical abnormalities in adulthood. Neuroscience Research, 64, 297. doi:10.1016/j.neures.2009.03.015

[22] Rana, S.A., Aavani, T. and Pittman, Q.J. (2012) Sex effects on neurodevelopmental outcomes of innate immune activation during prenatal and neonatal life. Hormones and Behavior, 62, 228-236.

[23] Ayhan, Y., Sawa, A., Ross, C.A. and Pletnikov, M.V. (2009) Animal models of gene-environment interactions in schizophrenia. Behavioural Brain Research, 204, 274281. doi:10.1016/j.bbr.2009.04.010

[24] Meyer, U., Nyffeler, M., Schwendener, S., Knuesel, I., Yee, B.K. and Feldon, J. (2008) Relative prenatal and postnatal maternal contributions to schizophrenia-related neurochemical dysfunction after in utero immune challenge. Neuropsychopharmacology, 33, 441-456.

\section{doi:10.1038/sj.npp.1301413}

[25] Miller, B.J., Culpepper, N., Rapaport, M.H. and Buckley, P. (2012) Prenatal inflammation and neurodevelopment in schizophrenia: A review of human studies. Progress in Neuro-Psychopharmacology and Biological Psychiatry, 42, 92-100.

[26] Piontkewitz, Y., Arad, M. and Weiner, I. (2011) Abnormal trajectories of neurodevelopment and behavior following in utero insult in the rat. Biological Psychiatry, 70, 842-851. doi:10.1016/j.biopsych.2011.06.007

[27] Kato, T., Abe, Y., Sotoyama, H., Kakita, A., Kominami, R., Hirokawa, S. and Nawa, H. (2010) Transient exposure of neonatal mice to neuregulin-1 results in hyperdopaminergic states in adulthood: Implication in neurodevelopmental hypothesis for schizophrenia. Molecular Psychiatry, 16, 307-320. doi:10.1038/mp.2010.10

[28] Erta, M., Quintana, A. and Hidalgo, J. (2012) Interleukin6 , a major cytokine in the central nervous system. International Journal of Biological Sciences, 8, 1254. doi:10.7150/ijbs.4679

[29] Weinberger, D.R. (1987) Implications of normal brain development for the pathogenesis of schizophrenia. Archives of General Psychiatry, 44, 660. doi:10.1001/archpsyc.1987.01800190080012

[30] McDade, T.W. (2003) Life history theory and the immune system: Steps toward a human ecological immunology. American Journal of Physical Anthropology, 122, 100-125. doi:10.1001/archpsyc.1987.01800190080012

[31] Brown, A.S. (2011) The environment and susceptibility to schizophrenia. Progress in Neurobiology, 93, 23-58. doi:10.1016/j.pneurobio.2010.09.003

[32] Giovanoli, S., Engler, H., Engler, A., Richetto, J., Voget, M., Willi, R. and Meyer, U. (2013) Stress in puberty unmasks latent neuropathological consequences of prenatal immune activation in mice. Science, 339, 1095-1099. doi:10.1126/science.1228261 